\title{
Electromagnetic Resonances of a Straight Wire on an Earth-Air Interface
}

\section{Citation}

Myers, John M., Sheldon S. Sandler, and Tai Tsun Wu. 2011. “Electromagnetic Resonances of a Straight Wire on an Earth-Air Interface." IEEE Trans. Antennas Propagat. 59, no. 9: 3154-3163.

\section{Published Version}

doi:10.1109/tap.2011.2161539

\section{Permanent link}

http://nrs.harvard.edu/urn-3:HUL.InstRepos:12701524

\section{Terms of Use}

This article was downloaded from Harvard University's DASH repository, and is made available under the terms and conditions applicable to Open Access Policy Articles, as set forth at http:// nrs.harvard.edu/urn-3:HUL.InstRepos:dash.current.terms-of-use\#OAP

\section{Share Your Story}

The Harvard community has made this article openly available.

Please share how this access benefits you. Submit a story.

\section{Accessibility}




\title{
Electromagnetic Resonances of a Straight Wire on an Earth-Air Interface
}

\author{
John M. Myers, Sheldon S. Sandler, and Tai Tsun Wu
}

\begin{abstract}
Using a variational method, we recently determined an electromagnetic "signature" for characterizing a straight wire in free space. The signature consists of the first five resonant frequencies and their widths, more compactly expressed as the first five complex-valued resonant frequencies. Here we apply the variational method to the much more complicated case of determining the same signature for a straight wire or wire pair on a flat interface between a homogeneous earth and air. To calculate the resonances we obtain an integral equation for the current on a wire on the interface between two dielectric media. Complex-valued resonant frequencies are defined as those for which the homogeneous integral equation for the current in an equivalent thin strip on the interface has non-zero solutions. The variational method extracts good approximations to these complex-valued resonant frequencies, without having to solve the integral equation. A table of resonances is given for the case of a relative dielectric constant of the earth equal to 4 and for three values of the ratio of wire radius to wire half-length.
\end{abstract}

\section{Index Terms}

Antenna theory, resonance, earth, interface phenomena, integral equation, variational methods.

\section{INTRODUCTION}

$\mathbf{I}$ $\mathrm{T}$ has long been of interest to establish electromagnetic signatures by which to recognize objects. Here we study complex-valued resonant frequencies [1], [2] in a straight wire located on the surface of a flat earth, to serve as a "signature" for locating such a wire. An important property of the resonances is that the ratios of successive resonant frequencies are characteristic of such a wire and are approximately invariant with wire diameter and wire length, so long as the diameter is very much less than both the wire length and the wavelength.

In the case of a thin wire in a homogeneous medium, the shape of its cross section has negligible effect on the electrical behavior of the wire, but some shapes are much more convenient than others for analysis. Although mostly interested in a wire of circular cross section, we draw on conformal mapping to find that for perfect conductors a circular wire of radius $a$ is essentially equivalent to a flat strip of width $4 a$ and negligible thickness [3]. This equivalence to a strip holds also for a thin wire on the boundary between two different dielectric media; indeed a wire pair can be treated the same way, namely as equivalent to a suitably scaled strip. We analyze a flat and perfectly conducting strip of zero thickness. We assume the strip is located in a plane interface, thought of as horizontal, between a medium below (earth) and a medium above (air). The problem is to find the first five resonant frequencies for electromagnetic radiation scattered by this thin strip as functions of the strip length, strip width, and the dielectric constant of earth and of air; the width of each resonance is also to be determined.

Our approach, based on the theory of linear antennas, is to formulate an integral equation for the current in the wire induced by an incident electromagnetic field. For the much simpler case of a wire in a single

This work was supported in part by the Army Research Office under Grant W911NF-07-1-0509 with Harvard University. The authors are with the Harvard School of Engineering and Applied Sciences, Cambridge, MA 02138 USA (e-mail: myers@seas.harvard.edu; sandler@seas.harvard.edu; ttwu@seas.harvard.edu). 
homogeneous medium, two forms of integral equation for the current generated by an incident electromagnetic field were well developed over fifty years ago [4]. The formulation of the Hallén integral equation for the current in the wire induced by an incident vector potential evades non-integrable singularities that would infest the integral if the integral equation were formulated directly for an incident electric field. From this Hallén integral equation, in which the vector potential plays a central role, it follows that the current on a thin antenna is approximately sinusoidal, and the propagation constant for this approximately sinusoidal current is that of the surrounding medium.

In the early 1970's resonant frequencies were defined as the complex values of frequency at which a homogeneous integral equation (in which the incident field is set to zero) has a non-zero solution for the current [1], [2]. For a straight wire thicker than that of interest to us, the Singularity Expansion Method (SEM) yielded solutions for the case of a homogeneous medium [5]. Recently we recast the SEM method to express the resonant frequencies as solutions to an equation that sets to zero a functional of the current, with the nice property that this functional has zero first-order variation under variation of the current [6]. This insensitivity allows a relatively crude approximation to the current at resonance to be used in solving for an accurate approximation to the complex-valued resonant frequency.

In contrast to the case of a wire in a single medium, for the present case of a wire on an interface, there is no useful definition of a vector potential, which complicates formulating the problem. When the wire involves two media with distinct propagation constants, the questions arise:

1) Is the current along the wire approximately sinusoidal?

2) If so, what is its propagation constant?

Based on the known expression for the electric field emanating from a point current element located on and parallel with the interface [7], it turns out that integral equations of both the Pocklington type and the Hallén type can be formulated, but with a more complex kernel that introduces qualitatively new features, associated physically with the complexity of paths by which energy can propagate near an interface. Solving the integral equation in any form is difficult; however, we will find a good approximation to the resonant values of $k h$ without having to solve the integral equation. As described below, the current near resonance is approximately sinusoidal, but with a complex-valued propagation constant, even when both media are lossless. Using the approximate current, the variational method developed for free space is applied to determine approximately the first five complex-valued resonant frequencies of a wire on an earth-air interface.

The rest of the paper is organized as follows.

1) In Sec. II, we state the known expression of the electric field generated by a current element on the strip. This electric-field kernel, however, is non-integrable and so cannot serve directly as the kernel of an integral equation.

2) To obtain an integrable kernel, the trick is similar to that used in the case of a single medium: in Sec. III we determine a pair of complex-valued zeros in the Fourier transform of the electric-field kernel with respect to distance along the strip, based on an approximation valid for a thin strip.

3) Using the zeros determined in Sec. III, in Sec. IV, we derive the integral equation of the Pocklington type satisfied by the current induced in the strip, and show how the values of the zeros in the Fourier transform of the electric field supply the main ingredient in the answer to the two questions posed above.

4) In Sec. V, the complex-valued resonant frequencies are defined as the frequencies at which the homogeneous integral equation for the current in the strip has non-zero solutions. The integral equation is manipulated into a form in which the solutions for the resonances are insensitive to small errors in the current, allowing us to use the kernel of the integral equation, obtained after much labor, together with a simple approximation for the current to obtain approximate equations for the resonant frequencies of better accuracy than the approximation for the current.

5) In Sec. VI, the equations defining the kernel of the integral equation for the current are rearranged to facilitate numerical computation, and examples of calculated resonances are reported.

6) In Sec. VII we make some concluding remarks. 


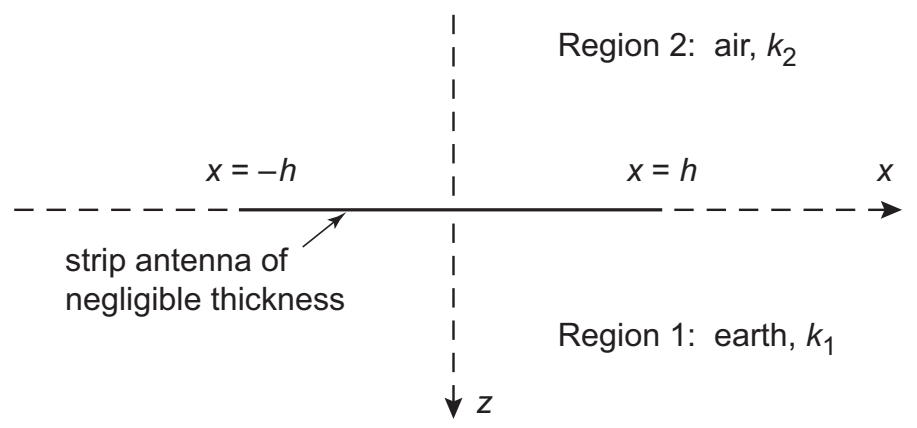

(a) side view

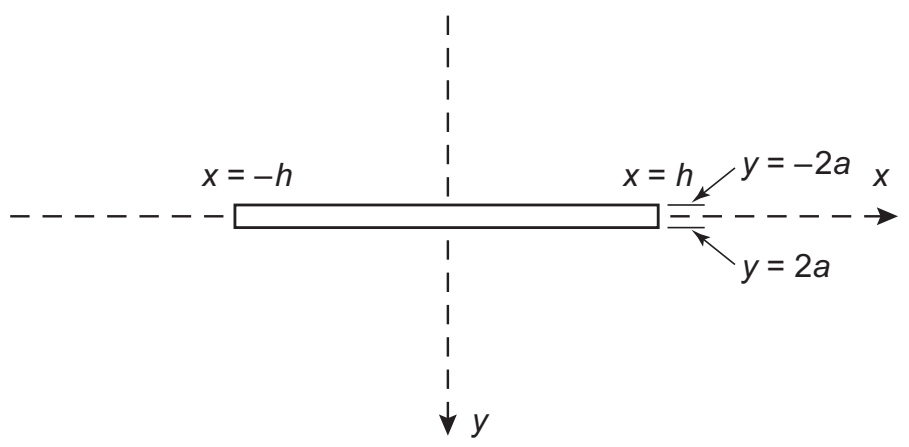

(b) top view

Fig. 1. Schematic diagram of the antenna geometry.

\section{FORMULATION}

We analyze a flat, perfectly conducting strip of length $2 h$, width $4 a$, and zero thickness, located in a plane interface, thought of as horizontal, between a medium below (earth) with a propagation constant $k_{1}$ and a medium above (air) with a propagation constant $k_{2}$, as shown schematically in Fig. 1. Following the notation of Ref. [7], let the planar boundary between air and earth be the $x y$-plane given by $z=0$, and let the $z$-axis point down into the direction of the earth. The problem is to find the first five complex resonant frequencies for electromagnetic radiation scattered by this thin strip as functions of the parameters $a, h$, $k_{1}$, and $k_{2}$.

As holds for earth and air, it will be assumed throughout this paper that

$$
k_{1}>k_{2} \text {. }
$$

Since the antenna is assumed to be thin, $a$ is taken to be small, so that

$$
a \ll h \text { and } k_{1} a \ll 1,
$$

which, of course, implies $k_{2} a \ll 1$. For present purposes both earth and air are assumed lossless, so that both $k_{1}$ and $k_{2}$ are taken to be real-valued and positive. (As in the usual case of the linear antenna in a homogeneous medium, once the lossless case is understood, the introduction of loss in the medium is fairly straightforward.)

The formulation of the problem hinges on the electric field generated by a "horizontal" point dipole in the interface. Consider a delta-function current at the origin,

$$
\mathbf{J}=\delta(x) \delta(y) \delta(z) \hat{\mathbf{x}}
$$

then, the $x$-component of the electric field at a point $(x, y, 0)$ on the interface is given by (see (5.4.13) of Ref. [7])

$$
E_{x}(x, y)=-\frac{\omega \mu_{0}}{4 \pi^{2}} \int_{-\infty}^{\infty} d \xi \int_{-\infty}^{\infty} d \eta e^{i(\xi x+\eta y)} \frac{\gamma_{1}\left(k_{2}^{2}-\xi^{2}\right)+\gamma_{2}\left(k_{1}^{2}-\xi^{2}\right)}{M N}
$$


where

$$
\begin{array}{ll}
\gamma_{1}=\left(k_{1}^{2}-\xi^{2}-\eta^{2}\right)^{1 / 2}, & \gamma_{2}=\left(k_{2}^{2}-\xi^{2}-\eta^{2}\right)^{1 / 2}, \\
M=\gamma_{1}+\gamma_{2}, & N=k_{1}^{2} \gamma_{2}+k_{2}^{2} \gamma_{1} .
\end{array}
$$

In deriving (4), it has been assumed that the magnetic permeability for both region 1 and region 2 is given by $\mu_{0}$; that is, we do not deal here with magnetizable earth. The advantage of studying a strip antenna of negligible thickness is the invariance of (4) under interchange of regions 1 and 2, unlike the much more complex formula that would express a thick wire.

At points off the strip, the electric field produced by the current density on the strip is expressed as an integral over $E_{x}\left(x-x^{\prime}, y-y^{\prime}\right)$ times the current density as a function of $x^{\prime}$ and $y^{\prime}$, so that $E_{x}(x, y)$ can be thought of as an "electric-field kernel"; however at points on the strip the integral fails to converge. As in the case of a single-medium, for the wire on an interface the key property of the electric-field kernel that makes possible the elimination of a non-integrable singularity is a pair of zeros of the spatial Fourier transform of the electric-field kernel.

In the single-medium case, the zeros are at $\pm k$ where $k$ is the propagation constant of the single medium, so the Fourier transform of the kernel has a factor $\left(\xi^{2}-k^{2}\right)$ which leads in the Pocklington integro-differential equation to the differential operator $\left(d^{2} / d x^{2}+k^{2}\right)$. That method works also for a thin strip at an interface, except that finding the zeros in the Fourier transform of $E_{x}$ is a considerable task, to which we now turn.

\section{ZEROS IN THE FOURIER TRANSFORM OF $E_{x}$}

From (4) it follows that the Fourier transform from $x$ to $\xi$ of $E_{x}(x, y)$ is $-\omega \mu_{0} / 2 \pi$ times

$$
\tilde{E}_{x}(\xi, y) \stackrel{\text { def }}{=} \int_{-\infty}^{\infty} d \eta e^{i \eta y} \frac{\gamma_{1}\left(k_{2}^{2}-\xi^{2}\right)+\gamma_{2}\left(k_{1}^{2}-\xi^{2}\right)}{M N} .
$$

This $\tilde{E}_{x}(\xi, y)$ depends also on $k_{1}$ and $k_{2}$, which in turn depend on frequency through

$$
k_{1}=\sqrt{\epsilon_{1}} \omega / c \text { and } k_{2}=\sqrt{\epsilon_{2}} \omega / c,
$$

where $\epsilon_{1}$ and $\epsilon_{2}$ are the relative dielectric constants of the two media. We assume lossless media, so that both $\epsilon_{1}$ and $\epsilon_{2}$ are real-valued and positive; taking region 2 to be air, $\epsilon_{2} \approx 1$. In marked contrast to the case of a single medium, however, the values of $\xi$ at which $\tilde{E}_{x}(\xi, y)$ is zero turn out to be complex, not real.

To find the zeros of $\tilde{E}_{x}(\xi, y)$, the integral on the right-hand side of (6) must be evaluated, which presents two difficulties: (a) this integral cannot be expressed exactly in terms of standard special functions, and (b) the integrand oscillates and decays only slowly, making it unsuitable for direct numerical evaluation. To overcome these difficulties, we develop approximations of the integral, accurate for a thin strip. Because $|y|<2 a$, and taking $y$ to be positive without loss of generality, the condition for the strip to be thin is

$$
k_{1} y \ll 1 \text { and }|\xi| y \ll 1 \text {. }
$$

The approximation to be developed has the form of the first two terms of a series expansion. Although it neglects terms of the order of $(\bar{\xi} y)^{2}|\ln (\bar{\xi} y)|$, where $\bar{\xi}$ is the larger one of $k_{1}$ and $|\xi|$, the approximation is highly accurate for small values of $y$.

To proceed, we rewrite (6) as

$$
\tilde{E}_{x}(\xi, y)=F_{1}(\xi, y)-\xi^{2} F_{2}(\xi, y),
$$

where

and

$$
F_{1}(\xi, y)=\int_{-\infty}^{\infty} d \eta e^{i \eta y} \frac{1}{\gamma_{1}+\gamma_{2}}
$$

$$
F_{2}(\xi, y)=\int_{-\infty}^{\infty} d \eta e^{i \eta y} \frac{1}{k_{1}^{2} \gamma_{2}+k_{2}^{2} \gamma_{1}}
$$


As discussed in detail in [8], $F_{1}(\xi, y)$ can be evaluated exactly in terms of Bessel functions. Then the small-argument approximation [9] for the Bessel functions together with an approximate evaluation of $F_{2}(\xi, y)$ described in [8] imply

$$
\begin{aligned}
\tilde{E}_{x}(\xi, y) & \frac{\pi}{2}+i\left\{\frac{1}{k_{1}^{2}-k_{2}^{2}}\left[\left(k_{1}^{2}-\xi^{2}\right) \ln \left(y \sqrt{k_{1}^{2}-\xi^{2}}\right)-\left(k_{2}^{2}-\xi^{2}\right) \ln \left(y \sqrt{k_{2}^{2}-\xi^{2}}\right)\right]+\gamma-\ln 2-\frac{1}{2}\right\} \\
& -\frac{\xi^{2}}{k_{1}^{2}+k_{2}^{2}}\left\{\pi+2 i\left[\frac{1}{k_{1}^{2}-k_{2}^{2}}\left(k_{1}^{2} \ln \left(y \sqrt{k_{2}^{2}-\xi^{2}}\right)-k_{2}^{2} \ln \left(y \sqrt{k_{1}^{2}-\xi^{2}}\right)+\gamma-\ln 2\right]\right.\right. \\
& -\frac{i k_{1}^{2} k_{2}^{2}}{k_{1}^{2}-k_{2}^{2}} \frac{1}{\sqrt{\left(k_{1}^{2}+k_{2}^{2}\right) \xi^{2}-k_{1}^{2} k_{2}^{2}}} \\
& \left.\times\left[\ln \frac{k_{1}^{2}+\sqrt{\left(k_{1}^{2}+k_{2}^{2}\right) \xi^{2}-k_{1}^{2} k_{2}^{2}}}{k_{1}^{2}-\sqrt{\left(k_{1}^{2}+k_{2}^{2}\right) \xi^{2}-k_{1}^{2} k_{2}^{2}}}-\ln \frac{k_{2}^{2}+\sqrt{\left(k_{1}^{2}+k_{2}^{2}\right) \xi^{2}-k_{1}^{2} k_{2}^{2}}}{k_{2}^{2}-\sqrt{\left(k_{1}^{2}+k_{2}^{2}\right) \xi^{2}-k_{1}^{2} k_{2}^{2}}}\right]\right\} .
\end{aligned}
$$

This is the desired approximate expression from which to determine the zero of this $\tilde{E}_{x}$ as a function of $\xi$ when $k_{1}, k_{2}$ and $y$ are given subject to the conditions (8). For several values of $k_{2} a$ and $k_{1} / k_{2}$, values of $k_{0}^{2} / k_{2}^{2}$ at which $\tilde{E}_{x}(\xi, y)$ as specified in (12) has a zero at $\xi=k_{0}$ are listed in Table I. Equation (12) determines a unique value for $k_{0}^{2}$, and hence a pair of values for $\pm k_{0}$; by $k_{0}$ we denote the complex value

\begin{tabular}{|c|c|c|c|c|c|}
\hline$k_{2} a$ & $k_{1} / k_{2}$ & $k_{0}^{2} / k_{2}^{2}$ & $k_{2} a$ & $k_{1} / k_{2}$ & $k_{0}^{2} / k_{2}^{2}$ \\
\hline $10^{-4}$ & 2.0 & $2.59013+i 0.16384$ & $10^{-4}$ & 2.2 & $3.04044+i 0.20489$ \\
\hline $10^{-5}$ & 2.0 & $2.56777+i 0.13092$ & $10^{-5}$ & 2.2 & $3.01066+i 0.16348$ \\
\hline $10^{-6}$ & 2.0 & $2.55413+i 0.10890$ & $10^{-6}$ & 2.2 & $2.99250+i 0.13586$ \\
\hline
\end{tabular}
having a positive real part.

TABLE I

SAMPLE VALUES OF $k_{0}^{2} / k_{2}^{2}$ AS A FUNCTION OF $k_{2} a$ AND $k_{1} / k_{2}$

\begin{tabular}{|c|c|c|}
\hline$k_{2} a$ & $k_{1} / k_{2}$ & $k_{0}^{2} / k_{2}^{2}$ \\
\hline $10^{-4}$ & 1.8 & $2.18318+i 0.12600$ \\
\hline $10^{-5}$ & 1.8 & $2.16737+i 0.10080$ \\
\hline $10^{-6}$ & 1.8 & $2.15777+i 0.08392$ \\
\hline
\end{tabular}

In this calculation the neglect of terms on the order of $\left|k_{1} a\right|^{2} \ln \left|k_{1} a\right|$ generates a relative error in $k_{0}$ of order $\left|k_{1} a\right|^{2}$. For the $n$-th resonant frequency, we bound $\left|k_{1} a\right|$ by $\left|k_{1} a\right| \leq 2^{-1 / 2} n \pi a / h$. For $n \leq 5$ and $a / h \leq 10^{-4}$, the neglected terms are $O\left(10^{-6}\right)$, and under these conditions, which hold for Table I, we expect a relative error in $\left(k_{0} / k_{2}\right)^{2}$ less than $10^{-5}$.

To gain more physical insight than is visible in these calculated numbers, we keep only the leading term of order $\ln (\bar{\xi} y)$ and neglect all terms of order 1 on the right-hand side of (12) to obtain the rough approximation:

$$
\tilde{E}_{x}(\xi, y) \approx i\left(1-\frac{2 \xi^{2}}{k_{1}^{2}+k_{2}^{2}}\right) \ln (\bar{\xi} y) .
$$

Thus, in this rough approximation, the zero of $\tilde{E}_{x}$, called $k_{0}$, is given simply by the known formula [10], [11]

$$
k_{0}^{2} \approx \frac{k_{1}^{2}+k_{2}^{2}}{2}
$$

A more accurate approximation yields an additional interesting result. Because the right-hand side of (12) is complex in the sense of having both a real part and an imaginary part, setting $\tilde{E}_{x}$ to zero can be expected to lead to a solution $\xi$ that is complex, and such a zero off the real axis leads to many new phenomena. Ref. [8] displays the approximate value of the imaginary part of the zero, to show that both 


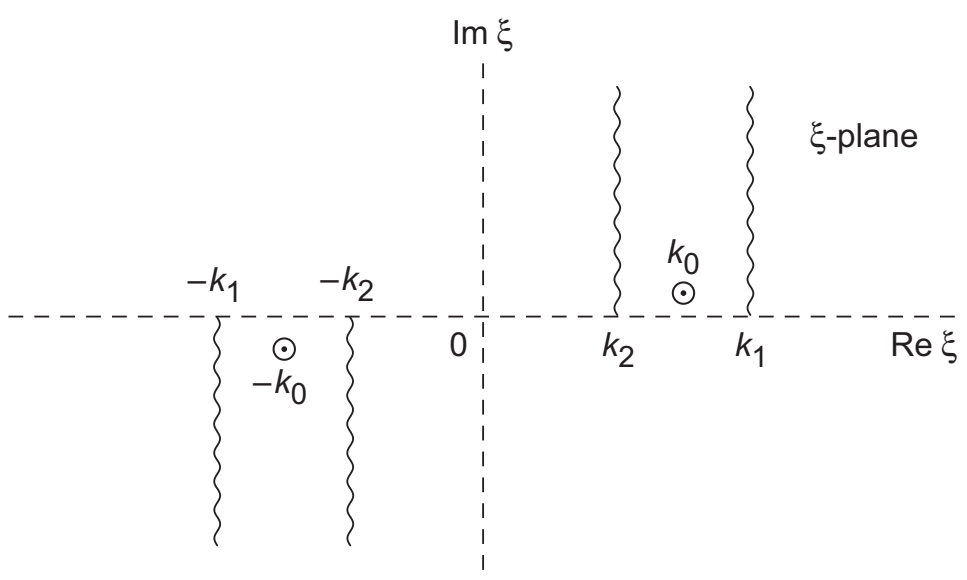

Fig. 2. A usual choice of the first ("proper") sheet in the $\xi$-plane. With this and a number of similar choices, the zeros of $\tilde{E}_{x}(\xi, y)$ at $\xi=k_{0}$ and $\xi=-k_{0}$ are on the first sheet.

the real and the imaginary parts of the zero point $k_{0}$ of $\tilde{E}_{x}(\xi, y)$ in the $\xi$-plane are positive when $k_{1}>k_{2}$, at least for small values of $y$. That $k_{0}$ is not real even in the absence of dissipation leads to features that are not seen in the usual case of the linear antenna in a uniform medium. Note that $k_{0}$ is in the first ("proper") sheet of the complex $\xi$-plane as usually chosen-see Fig. 2-unlike the Sommerfeld pole for the linear antenna.

\section{INTEGRAL EQUATION FOR THE CURRENT}

If the electric field kernel $E_{x}(x, y)$ had only an integrable singularity, it would work immediately as the kernel for an integral equation for the current in the wire; however because $E_{x}(x, y)$ can be shown to behave as $1 / x^{2}$ as $x$ tends toward zero, something has to be done to evade this non-integrable singularity. As in the case of a homogeneous medium, the trick is to express the non-integrable $E_{x}(x, y)$ as a differential operator acting on a function that has only a logarithmic singularity, and then to bring the differential operator outside the integration.

The needed differential operator is obtained by exploiting the zero-points $\pm k_{0}$ in the Fourier transform $\tilde{E}_{x}(\xi, y)$, found above, to put $E_{x}(x, y)$ into the form

$$
E_{x}(x, y)=\frac{\omega \mu_{0}}{4 \pi^{2}}\left(\frac{\partial^{2}}{\partial x^{2}}+k_{0}^{2}\right) G\left(k_{1}, k_{2}, x, y\right),
$$

where the function $G\left(k_{1}, k_{2}, x, y\right)$, which has only a logarithmic singularity, follows from defining its Fourier transform to be

$$
\tilde{G}\left(k_{1}, k_{2}, \xi, y\right)=\frac{\tilde{E}_{x}(\xi, y)}{\xi^{2}-k_{0}^{2}} .
$$

From (16) together with (6) one obtains (15). Inverting the Fourier transform $\tilde{G}\left(k_{1}, k_{2}, \xi, y\right)$ gives

$$
\begin{aligned}
G\left(k_{1}, k_{2}, x, y\right) & =\int_{-\infty}^{\infty} d \xi e^{i \xi x} \tilde{G}\left(k_{1}, k_{2}, \xi, y\right) \\
& =\int_{-\infty}^{\infty} \frac{d \xi}{\xi^{2}-k_{0}^{2}} \int_{-\infty}^{\infty} d \eta e^{i(\xi x+\eta y)} \frac{\gamma_{1}\left(k_{2}^{2}-\xi^{2}\right)+\gamma_{2}\left(k_{1}^{2}-\xi^{2}\right)}{M N} .
\end{aligned}
$$

For comparison, in the case of a homogeneous medium characterized by $k_{1}=k_{2}$, one finds [8]

$$
G\left(k_{1}, k_{1}, x, y\right)=\frac{-\pi i}{k_{1}^{2}} \frac{\exp \left(i k_{1} \sqrt{x^{2}+y^{2}}\right)}{\sqrt{x^{2}+y^{2}}} .
$$


The argument that a thin strip antenna of width $4 a$ is electromagnetically equivalent to a circular one of radius $a$ (see Ref. [4], pp. 16-18) works without change for the more general situation of two media, leading to the integro-differential equation

$$
\left(\frac{\partial^{2}}{\partial x^{2}}+k_{0}^{2}\right) \int_{-h}^{h} d x^{\prime} \boldsymbol{K}\left(x-x^{\prime}\right) I\left(x^{\prime}\right)=\frac{4 \pi}{\omega \mu_{0}} E^{\text {inc }}(x),
$$

where, from (15) and (17), the kernel $\boldsymbol{K}$ of the equation is

$$
\boldsymbol{K}(x)=\frac{1}{2 \pi} \int_{-\pi}^{\pi} d \theta G\left(k_{1}, k_{2}, x, 2 a \sin \frac{1}{2} \theta\right),
$$

$I$ is the current on the antenna, and $E^{\text {inc }}(x) \stackrel{\text { def }}{=} E_{x}^{\text {inc }}(x, 0)$ is the $x$-component of the external electric field applied to the thin strip, evaluated at $y=0$.

Equation (19) together with the approximation (14) to the zeros in the Fourier transform of $E_{x}(x, y)$ allows one to answer the two questions posed in the introduction. For a wire in free space there appears a factor $\left(d^{2} / d x^{2}+k^{2}\right)$ in the Pocklington equation, where $k$ is the free-space propagation constant, and this factor contributes large terms of the form $\cos k x$ when $k h$ is near $n \pi / 2$ for $n$ odd. Thus at resonance with $n$ odd, the current is roughly approximated by a constant times $\cos k x \approx \cos (n \pi x / 2 h)$. Similarly for $n$ even the current is roughly approximated by a constant times $\sin (n \pi x / 2 h)$. For the present case of a wire on an interface, the Pocklington-type equation (19) has the factor $\left(d^{2} / d x^{2}+k_{0}^{2}\right)$, with the result that near resonance the current is roughly approximated as in the free-space case except that $k$ of free space is replaced by the complex-value $k_{0} \approx \sqrt{\left(k_{1}^{2}+k_{2}^{2}\right) / 2}$.

\section{COMPleX-VALUED RESONANT FREQUENCIES}

As in [1], [2], we relate complex-valued resonant frequencies to solutions of the homogeneous integral equation, that is the integral equation (19) with $E^{\text {inc }}(x)$ on $-h \leq x \leq h$ set to 0 :

$$
\left(\frac{\partial^{2}}{\partial x^{2}}+k_{0}^{2}\right) \int_{-h}^{h} d x^{\prime} \boldsymbol{K}\left(x-x^{\prime}\right) I\left(x^{\prime}\right)=0 .
$$

At first glance, one might expect the only solution to be $I(x)=0$. However, the key to defining resonance is to note that the kernel $\boldsymbol{K}$ depends not only on position along the wire, but also on the angular frequency $\omega$, on which $k_{1}$ and $k_{2}$ depend through (7). Therefore the solution to the integral equation (21) depends on $\omega$, and for certain discrete special values of $\omega$, denoted $\omega_{1}, \omega_{2}, \ldots$, the equation has non-zero solutions. These values are expected to be complex, so that, listed in increasing order of their real parts, we have

$$
\begin{aligned}
\operatorname{Re} \omega_{n} & =\text { the } n \text {-th resonance frequency, } \\
-\operatorname{Im} \omega_{n} & =\text { the half-width at half maximum of the } n \text {-th resonance. }
\end{aligned}
$$

Under the assumption made here that $k_{2}$ is real-valued, we choose instead of $\omega_{n}$ the parameter $k_{2, n} h$ (where $k_{2, n}=\left(\omega_{n} / c\right) \sqrt{\epsilon_{2}}$, with $c$ the speed of light in vacuum) to express the $n$-th complex resonant frequency. The issue is how to find the $k_{2, n} h$ at which (21) has non-zero solutions.

\section{A. Approximate solution for resonant frequencies}

To determine the complex values $k_{2, n} h$, we use a variational technique which can be expressed symbolically as follows. Noting that for the $n$-th resonance, $k_{1}$ and $k_{0}$ both depend on $k_{2, n}$, we let $A\left(k_{2, n}\right)$ denote the linear integro-differential operator in (21), and let the current for the $n$-th resonance be denoted by $I_{n}\left(k_{2, n}, x\right)$ so that $(21)$ is abbreviated as

$$
A\left(k_{2, n}\right) * I_{n}\left(k_{2, n}\right)=0
$$


where the $*$ denotes an integral over the spatial variable, and we have omitted writing the spatial variables $x$ and $x^{\prime}$ to emphasize the dependence of $A$ and $I_{n}$ on the propagation constant $k_{2, n}$. The problem of resonance is to determine for $n=1, \ldots, 5$ the $k_{2, n}$ such that $A\left(k_{2, n}\right) * I_{n}\left(k_{2, n}\right)=0$ has a solution for non-zero $I_{n}\left(k_{2, n}\right)$. What we require is only $k_{2, n}$; we do not seek an accurate solution to the current $I_{n}\left(k_{2, n}\right) \equiv I_{n}\left(k_{2, n}, x\right)$.

As in the case of a wire in a homogeneous medium [6], the method for determining $k_{2, n}$ is based on considering the functional

$$
\begin{aligned}
S[I] & \stackrel{\text { def }}{=} I\left(k_{2, n}\right) * A\left(k_{2, n}\right) * I\left(k_{2, n}\right) \\
& =\int_{-h}^{h} d x I\left(k_{2, n}, x\right)\left(\frac{d^{2}}{d x^{2}}+\left[k_{0}\left(k_{2, n}\right)\right]^{2}\right) \int_{-h}^{h} d x^{\prime} \boldsymbol{K}\left(x-x^{\prime}\right) I\left(k_{2, n}, x^{\prime}\right) .
\end{aligned}
$$

Suppose that for some value $k_{2, n}$ there is a non-zero solution $I_{n}\left(k_{2, n}, x\right)$ to (21). As shown in [6], the variation with respect to $I$ (as a function of $x$ ) around this $I_{n}\left(k_{2, n}\right)$ of $S[I]$ is zero, that is:

$$
0=\delta\left[I_{n}\left(k_{2, n}\right) * A\left(k_{2, n}\right) * I_{n}\left(k_{2, n}\right)\right] .
$$

Equation (21) is just the statement that $A(k) * I_{n}(k)=0$, whence it follows that $I_{n}\left(k_{2, n}\right) * A\left(k_{2, n}\right) *$ $I_{n}\left(k_{2, n}\right)=0$. But since the first variation of the left-hand side is zero, replacing $I_{n}\left(k_{2, n}\right)$ by an approximation $I_{n}^{\text {ap }}$ makes no first-order error in the expression $I_{n}^{\text {ap }} * A\left(k_{2, n}\right) * I_{n}^{\text {ap }}$. Thus we will determine $k_{2, n}$ as the solution, for a suitable approximating current $I_{n}^{\text {ap }}$, to

$$
0=I_{n}^{\text {ap }} * A\left(k_{2, n}\right) * I_{n}^{\text {ap }} .
$$

Now we take advantage of the relative insensitivity of (24) to choose a simple approximating current. The resonances partition into those for which $n$ is odd and the current is symmetric in $x$ and those for which $n$ is even and the current is anti-symmetric in $x$. For a single medium, we discussed in [6] the "shifted-cosine" approximation to the current, and rejected it because it introduced spurious values for the resonant frequencies. As in that case, here for the case of two media we approximate the resonant current for $n$ odd as $I_{n}^{\text {ap }} \sim \cos (n \pi x / 2 h)$, and for $n$ even as $I_{n}^{\text {ap }} \sim \sin (n \pi x / 2 h)$.

For computational convenience we carry an $x$-derivative under the integral and integrate by parts to obtain for the equation for $k_{2, n}$

$$
0=\int_{-h}^{h} d x \int_{-h}^{h} d x^{\prime} \frac{d I_{n}^{\mathrm{ap}}(x)}{d x} \boldsymbol{K}\left(x-x^{\prime}\right) \frac{d I_{n}^{\mathrm{ap}}\left(x^{\prime}\right)}{d x^{\prime}}-k_{0}^{2} \int_{-h}^{h} d x \int_{-h}^{h} d x^{\prime} I_{n}^{\mathrm{ap}}(x) \boldsymbol{K}\left(x-x^{\prime}\right) I_{n}^{\mathrm{ap}}\left(x^{\prime}\right) ;
$$

the dependence on $k_{2, n}$, the sought value of $k_{2}$, is now limited to the dependence of the kernel $\boldsymbol{K}\left(x-x^{\prime}\right)$ and the parameter $k_{0}$ on $k_{2, n}$.

\section{B. Resonances}

Define $\kappa_{n} \stackrel{\text { def }}{=} n \pi / 2 h$. With the chosen approximating currents, (25) for determining $k_{2, n}$ for the case of symmetric currents in which $n$ is odd becomes

$$
\begin{aligned}
& \int_{-h}^{h} d x\left[-\frac{\partial}{\partial x} \cos \kappa_{n} x\right] \int_{-h}^{h} d x^{\prime} \boldsymbol{K}\left(x-x^{\prime}\right) \frac{\partial}{\partial x^{\prime}} \cos \kappa_{n} x^{\prime} \\
& \quad+k_{0}^{2} \int_{-h}^{h} d x \cos \kappa_{n} x \int_{-h}^{h} d x^{\prime} \boldsymbol{K}\left(x-x^{\prime}\right) \cos \kappa_{n} x^{\prime}=0 .
\end{aligned}
$$

We define

$$
\begin{gathered}
I_{s n} \stackrel{\text { def }}{=} \int_{-h}^{h} d x \sin \kappa_{n} x \int_{-h}^{h} d x^{\prime} \boldsymbol{K}\left(x-x^{\prime}\right) \sin \kappa_{n} x, \\
I_{c n} \stackrel{\text { def }}{=} \int_{-h}^{h} d x \cos \kappa_{n} x \int_{-h}^{h} d x^{\prime} \boldsymbol{K}\left(x-x^{\prime}\right) \cos \kappa_{n} x,
\end{gathered}
$$


and carry out the differentiations in (26) to obtain for $n$ odd:

$$
-\kappa_{n}^{2} I_{s n}+k_{0}^{2} I_{c n}=0,
$$

which in a form more convenient for calculation becomes

$$
\left(\kappa_{n}^{2}-k_{0}^{2}\right)\left(I_{s n}+I_{c n}\right)+\left(\kappa_{n}^{2}+k_{0}^{2}\right)\left(I_{s n}-I_{c n}\right)=0 .
$$

Similarly for the resonances with antisymmetric currents so that $n$ is even, one finds

$$
\begin{aligned}
& \int_{-h}^{h} d x\left[-\frac{\partial}{\partial x} \sin \kappa_{n} x\right] \int_{-h}^{h} d x^{\prime} \boldsymbol{K}\left(x-x^{\prime}\right) \frac{\partial}{\partial x^{\prime}} \sin \kappa_{n} x^{\prime} \\
& +k_{0}^{2} \int_{-h}^{h} d x \sin \kappa_{n} x \int_{-h}^{h} d x^{\prime} \boldsymbol{K}\left(x-x^{\prime}\right) \sin \kappa_{n} x^{\prime}=0
\end{aligned}
$$

which implies

$$
\left(\kappa_{n}^{2}-k_{0}^{2}\right)\left(I_{s n}+I_{c n}\right)-\left(\kappa_{n}^{2}+k_{0}^{2}\right)\left(I_{s n}-I_{c n}\right)=0 .
$$

To find the complex-valued resonant frequencies, one holds fixed the geometrical and material parameters $h, a, \epsilon_{1}, \epsilon_{2}$ and $\mu_{0}$ while varying the angular frequency $\omega$ in order to find complex values of $\omega$ for which (30) for the symmetric resonances and (32) for the antisymmetric resonances have non-zero solutions. Varying $\omega$ in the complex plane implies also varying the propagation constants $k_{1}, k_{2}$ via $k_{j}=\omega \sqrt{\epsilon_{j}} / c$ for $j=1,2$; and the dependence of $k_{1}$ and $k_{2}$ on (complex-valued) $\omega$ varies $k_{0}$ as determined by (12).

The next step, derived in Appendix A, is to reduce the double integrals to single integrals to obtain, for all resonances, regardless of whether $n$ is even or odd,

$$
\left(\kappa_{n}^{2}-k_{0}^{2}\right) I_{\Sigma n}=\left(\kappa_{n}^{2}+k_{0}^{2}\right) I_{\Delta n}
$$

where we define

$$
\begin{aligned}
& I_{\Sigma n} \stackrel{\text { def }}{=} I_{s n}+I_{c n}=2 \int_{0}^{2 h} d x \boldsymbol{K}(x)(2 h-x) \cos \kappa_{n} x, \\
& I_{\Delta n} \stackrel{\text { def }}{=}(-1)^{n}\left(I_{s n}-I_{c n}\right)=\frac{2}{\kappa_{n}} \int_{0}^{2 h} d x \boldsymbol{K}(x) \sin \kappa_{n} x .
\end{aligned}
$$

\section{Scaling to $h=1$}

For calculational purposes, we save effort by recognizing that the resonant frequencies scale with the wire half-length $h$. Thus one can set $h$ to 1 and obtain resonant frequencies for other values of $h$ by dividing by $h$. With $h=1$ we have $\kappa_{n}=n \pi / 2$ and the task is to solve the equation for resonances, namely, regardless of whether $n$ is even or odd,

$$
\left[(n \pi / 2)^{2}-k_{0}^{2}\right] I_{\Sigma n}=\left[(n \pi / 2)^{2}+k_{0}^{2}\right] I_{\Delta n},
$$

where with $h=1$ and $\kappa_{n}=n \pi / 2$ we have

$$
\begin{aligned}
& I_{\Sigma n} \stackrel{\text { def }}{=} I_{s n}+I_{c n}=2 \int_{0}^{2} d x \boldsymbol{K}(x)(2-x) \cos \frac{n \pi x}{2}, \\
& I_{\Delta n} \stackrel{\text { def }}{=}(-1)^{n}\left(I_{s n}-I_{c n}\right)=\frac{4}{n \pi} \int_{0}^{2} d x \boldsymbol{K}(x) \sin \frac{n \pi x}{2} .
\end{aligned}
$$

Similar to the case of the wire in a homogeneous medium, when the strip width $4 a$ is much smaller than all the other dimensions in this scattering problem, we can approximate the kernel $\boldsymbol{K}(x)$ [defined in (20)] by $G\left(k_{1}, k_{2}, x, a\right)$ defined in (17), so that we have

$$
\begin{aligned}
\boldsymbol{K}(x) & \approx G\left(k_{1}, k_{2}, x, a\right) \\
& =\int_{-\infty}^{\infty} \frac{d \xi}{\xi^{2}-k_{0}^{2}} \int_{-\infty}^{\infty} d \eta e^{i(\xi x+\eta a)} \frac{\gamma_{1}\left(k_{2}^{2}-\xi^{2}\right)+\gamma_{2}\left(k_{1}^{2}-\xi^{2}\right)}{M N} .
\end{aligned}
$$

We will use this approximation to determine resonances. 


\section{Accuracy}

Apart from the minor contribution of numerical integrations to error, the error in the complex-valued resonant frequencies comes from the neglected terms in the solution for the pole in the Fourier transform of the electric-field kernel (Sec. III), the approximation of the kernel by (37), and the inexactitude of the approximation to the resonant current. For thin wires with $\left|k_{1} a\right|<10^{-3}$, by far the largest contribution to error in the complex-valued resonant frequency is the inexactitude of approximation to the current. In order to get an idea of the size of this error, we proceed in a two-phase cycle: first we provisionally accept the value of complex-valued resonant frequency $k_{2, n}$, which we now rewrite as $k_{2, n}^{(1)}$, determined as above and use it to obtain an improved approximation $I^{\mathrm{ap}(2)}(x)$ to the current; second we use the improved approximation to the current to refine the approximation of the resonant frequency, denoted $k_{2, n}^{(2)}$. Although here we limit ourselves to a single round of the cycle, the iteration can be continued.

1) Phase I: We view the dielectric constant as fixed, so that $k_{1}$ is rigidly geared to $k_{2}$. Making explicit the dependence of the kernel and the current on the parameter $k_{2}$, we have that for $n$ odd and $k_{2}$ evaluated at the complex resonant frequency $k_{2, n}$, the exact current $I\left(k_{2} ; x\right)$ solves the equation obtained by transforming (21) and imposing a normalization condition:

$$
\int_{-h}^{h} d x^{\prime} \boldsymbol{K}\left(k_{2}, x-x^{\prime}\right) I\left(k_{2} ; x^{\prime}\right)-\cos \left(k_{0} x\right)=0
$$

(for $n$ even the cosine is replaced by a sine). When the approximate current $I^{\text {ap }}(x)$ is substituted for the exact current $I\left(k_{2} ; x\right)$, the equation no longer holds, and the discrepancy can be expressed by

$$
\chi\left[k_{2} ; I^{\text {ap }}\right] \stackrel{\text { def }}{=}\left[\int_{-h}^{h} d x\left|\int_{-h}^{h} d x^{\prime} K\left(k_{2} ; x-x^{\prime}\right) I^{a p}(x)-\cos \left(k_{0} x\right)\right|^{2}\right]^{1 / 2} .
$$

To get a better approximation of the current, $I^{\text {ap }(2)}(x)$, we allow additional terms in the approximating current, with coefficients obtained by minimizing the resulting $\chi\left[k_{2} ; I^{\text {ap }(2)}\right]$ evaluated at $k_{2}=k_{2, n}^{(1)}$.

2) Phase II: Insert the $I^{\mathrm{ap}(2)}(x)$ determined in Phase I into (25) to compute $k_{2, n}^{(2)}$. Reduction in error is measured by the ratio

$$
\frac{\left.\chi\left[k_{2} ; I^{\mathrm{ap}(2)}\right]\right|_{k_{2}=k_{2, n}^{(2)}}}{\left.\chi\left[k_{2} ; I^{\mathrm{ap}}\right]\right|_{k_{2}=k_{2, n}^{(1)}}}
$$

Result for lowest resonance in free space: For the lowest resonance $(n=1)$ in free space $(\epsilon=1)$ we carried out the above steps. To get a better approximation to the current we enlarge the space of possible currents to include not only the term $\cos (\pi x / 2 h)$ in the approximating current but also a term $\cos (3 \pi x / 2 h)$, so the approximating current becomes

$$
I^{\operatorname{ap}(2)}(x)=A[\cos (\pi x / 2 h)+B \cos (3 \pi x / 2 h)],
$$

where $A$ and $B$ are determined numerically by minimizing $\chi\left[k_{2, n}^{(1)} ; I^{\mathrm{ap}(2)}\right]$.

For this case, with $a / h=10^{-4}$, we find $A=0.054767-i 0.00550$ and $B=-0.01487-i 0.000271$. The coefficient $A$ is just a normalization factor, while the coefficient $B$ gives the relative size of the correction to the approximating current; thus that correction is about $1.5 \%$. The error measure $\chi\left[I^{\mathrm{ap}(2)}\right]$ is reduced by a factor of 47 relative to $\chi\left[I^{\mathrm{ap}}\right]$. The resulting value for the free-space resonant frequency is $1.520849-i 0.066800$. The relative magnitude of the difference between this and the complex value $1.522174-i 0.0066372$ computed in [6] is a little less than $0.1 \%$. Because this first cycle of the iterative procedure reduces the error measure so strongly, we take $0.1 \%$ to be a reasonable estimate of the error in the complex-valued resonant frequency computed using $I^{\text {ap }}(x)$. We expect more or less the same size of error for the wire on the interface, and we expect less error for smaller values of $a / h$; determining the error for higher resonances $(n>1)$ is left to future work. 


\section{NUMERICAL ANALYSIS OF RESONANCES}

We numerically solved (35) through (37) with $\boldsymbol{K}(x)$ approximated by (37) for a wire scaled to a half-length of $h=1$. To do this we had to evade two obstacles.

The first obstacle is that, for complex-valued resonant frequencies of interest, the integration path must be deformed to avoid crossing branch cuts. For keeping track of branch cuts, we note that for imaginary values of the parameters $k_{0}, k_{1}$, and $k_{2}$, the kernel $\boldsymbol{K}(x)$ is real-valued. For this reason, keeping track of branch cuts is made easier by changing variables to $\beta$ 's and the corresponding $\tilde{\gamma}$ 's defined by

$$
\begin{array}{rr}
(\text { For } j=0,1,2) & k_{j}=i \beta_{j}, \\
(\text { For } j=1,2) & \gamma_{j}=i \tilde{\gamma}_{j},
\end{array}
$$

where

$$
\tilde{\gamma}_{j} \stackrel{\text { def }}{=} \sqrt{\xi^{2}+\eta^{2}+\beta_{j}^{2}} \text {. }
$$

For $M$ and $N$ defined in (5) we have

$$
M=i\left(\tilde{\gamma}_{1}+\tilde{\gamma}_{2}\right) ; \quad N=-i\left(\beta_{2}^{2} \tilde{\gamma}_{1}+\beta_{1}^{2} \tilde{\gamma}_{2}\right) .
$$

For real values of $\beta$ 's, the branch points and branch lines present no trouble. We determine the proper branches involved in various integrals by following a path from positive real $\beta$ 's to whatever complex values we want with the rule of "no cross branch lines" which tells us how to deform branch lines so as to avoid such crossings. To express the kernel $\boldsymbol{K}(x)$ given in (37), we define

$$
\tilde{M}=\tilde{\gamma}_{1}+\tilde{\gamma}_{2} ; \quad \tilde{N}=\beta_{2}^{2} \tilde{\gamma}_{1}+\beta_{1}^{2} \tilde{\gamma}_{2} .
$$

After dropping constants that cancel out in the equations for resonant frequencies, for real-valued frequencies the kernel of the integral equation can be taken to be

$$
\begin{aligned}
\boldsymbol{K}(x) & =\int_{-\infty}^{\infty} \frac{d \xi}{\xi^{2}+\beta_{0}^{2}} \int_{-\infty}^{\infty} d \eta e^{i(\xi x+\eta a)} \frac{\tilde{\gamma}_{1}\left(\xi^{2}+\beta_{2}^{2}\right)+\tilde{\gamma}_{2}\left(\xi^{2}+\beta_{1}^{2}\right)}{\left(\tilde{\gamma}_{1}+\tilde{\gamma}_{2}\right)\left(\beta_{2}^{2} \tilde{\gamma}_{1}+\beta_{1}^{2} \tilde{\gamma}_{2}\right)} \\
& =\int_{-\infty}^{\infty} \frac{d \xi}{\xi^{2}+\beta_{0}^{2}} \int_{-\infty}^{\infty} d \eta e^{i(\xi x+\eta a)}\left(\frac{1}{\tilde{M}}+\frac{\xi^{2}}{\tilde{N}}\right) .
\end{aligned}
$$

The kernel $\boldsymbol{K}(x)$ depends on the resonant frequency through its dependence on $\beta_{1}, \beta_{2}$, and $\beta_{0}$, where $\beta_{0}$ itself is defined by the relation of $k_{0}$ to $k_{1}$ and $k_{2}$ given in Sec. III.

The integrand has poles at $\xi= \pm i \beta_{0}$. In [8] we show that for a real-valued dielectric constant $\epsilon=4$, over the range $0 \leq \alpha \leq 1.8, e^{i \alpha} \beta_{0}$ has a relatively large positive real part and a small positive imaginary part for the pole that contributes to contour integrals evaluated in [8].

To avoid direct integration over an oscillating integrand that drops off only slowly, we split the integrand into a part that drops off slowly but can be integrated exactly analytically and other parts that have to be integrated numerically but that converge faster. By use of

$$
\frac{1}{\tilde{M}}+\frac{\xi^{2}}{\tilde{N}}=\frac{\xi^{2}+\beta_{0}^{2}}{\beta_{1}^{2}+\beta_{2}^{2}} \frac{2}{\tilde{M}}+\left(1-\frac{2 \beta_{0}^{2}}{\beta_{1}^{2}+\beta_{2}^{2}}\right) \frac{1}{\tilde{M}}+\frac{\xi^{2}}{\beta_{1}^{2}+\beta_{2}^{2}}\left(\frac{\beta_{1}^{2}+\beta_{2}^{2}}{\tilde{N}}-\frac{2}{\tilde{M}}\right),
$$

one obtains

$$
\boldsymbol{K}(x)=\boldsymbol{K}_{1}(x)+\boldsymbol{K}_{2}(x)+\boldsymbol{K}_{3}(x),
$$

where

$$
\begin{aligned}
\boldsymbol{K}_{1}(x) & =\frac{2}{\beta_{1}^{2}+\beta_{2}^{2}} \int_{-\infty}^{\infty} d \xi \int_{-\infty}^{\infty} d \eta e^{i(\xi x+\eta a)} \frac{1}{\tilde{M}} \\
\boldsymbol{K}_{2}(x) & =\left(1-\frac{2 \beta_{0}^{2}}{\beta_{1}^{2}+\beta_{2}^{2}}\right) \int_{-\infty}^{\infty} \frac{d \xi}{\xi^{2}+\beta_{0}^{2}} \int_{-\infty}^{\infty} d \eta e^{i(\xi x+\eta a)} \frac{1}{\tilde{M}},
\end{aligned}
$$




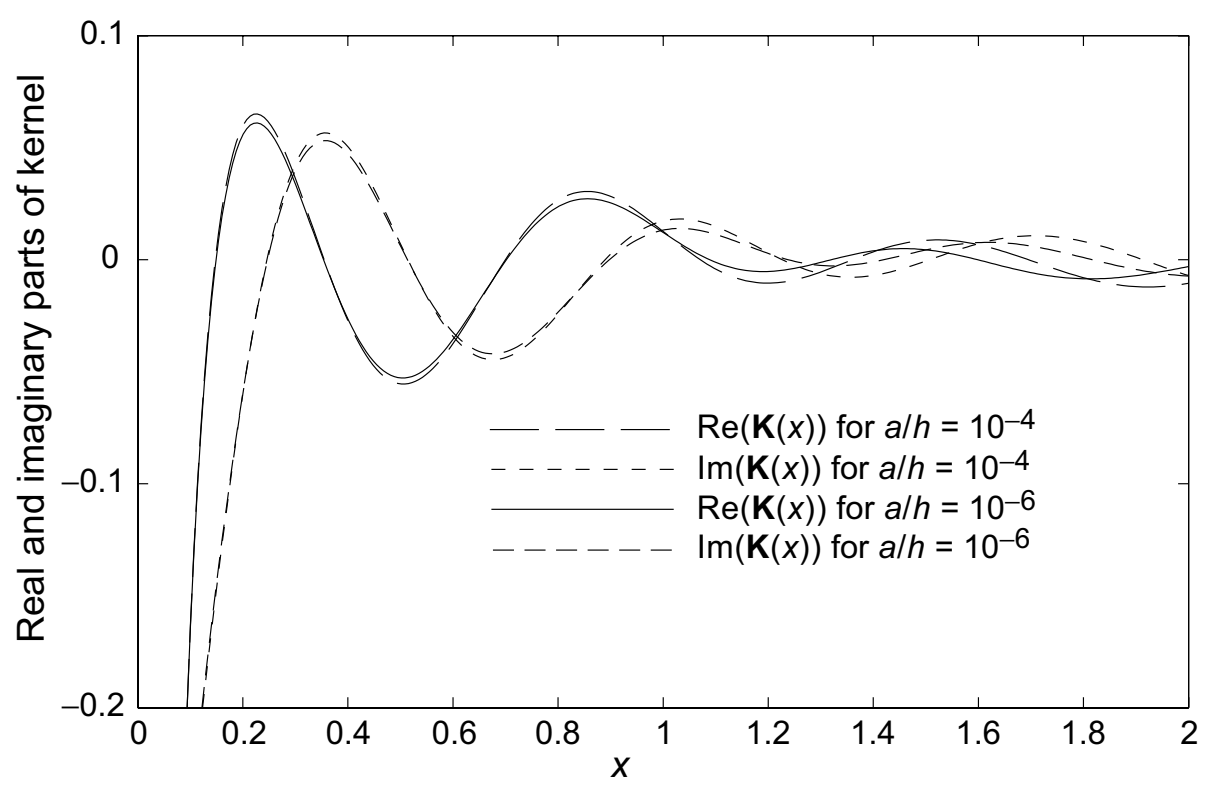

Fig. 3. Two cases of the kernel $\mathbf{K}(x)$ when $k_{1} / k_{2}=2$ and $k_{2} h=5 ; a / h=10^{-4}$ and $a / h=10^{-6}$.

and

$$
\begin{aligned}
\boldsymbol{K}_{3}(x) & =\frac{1}{\beta_{1}^{2}+\beta_{2}^{2}} \int_{-\infty}^{\infty} d \xi \frac{\xi^{2}}{\xi^{2}+\beta_{0}^{2}} \int_{-\infty}^{\infty} d \eta e^{i(\xi x+\eta a)}\left(\frac{\beta_{1}^{2}+\beta_{2}^{2}}{\tilde{N}}-\frac{2}{\tilde{M}}\right) \\
& \approx \frac{1}{\beta_{1}^{2}+\beta_{2}^{2}} \int_{-\infty}^{\infty} d \xi e^{i \xi x} \frac{\xi^{2}}{\xi^{2}+\beta_{0}^{2}} \int_{-\infty}^{\infty} d \eta\left(\frac{\beta_{1}^{2}+\beta_{2}^{2}}{\tilde{N}}-\frac{2}{\tilde{M}}\right) .
\end{aligned}
$$

The last relation follows because the integrand falls off fast enough as $\eta$ becomes large to make $\boldsymbol{K}_{3}(x)$ smooth as $x \rightarrow 0$, so that in the integrand we replace $a$ by 0 without significant loss of accuracy.

The term $\boldsymbol{K}_{1}(x)$ can be evaluated exactly [8]:

$$
\boldsymbol{K}_{1}(x)=\frac{4 \pi}{\left(\beta_{1}^{4}-\beta_{2}^{4}\right) \rho^{3}}\left[\left(1+\beta_{2} \rho\right) e^{-\beta_{2} \rho}-\left(1+\beta_{1} \rho\right) e^{-\beta_{1} \rho}\right]
$$

where $\rho \stackrel{\text { def }}{=} \sqrt{x^{2}+a^{2}}$. In [8] the integrals in $\boldsymbol{K}_{2}(x)$ and $\boldsymbol{K}_{3}(x)$ are transformed to single integrals and put into forms convenient for numerical evaluation.

Figure 3 shows the kernel $\boldsymbol{K}(x)$ for the case $k_{1} / k_{2}=2$ and $k_{2} h=5$, and for two values of $a / h=10^{-4}$ and $10^{-6}$. Unlike the wire in a homogeneous medium, the kernels for the two cases of $a / h$ differ noticeably even for values of $x \gg a$.

For the case of earth having a dielectric constant 4 times that of free space, leading to $k_{1} / k_{2}=2$, the first five normalized complex-valued resonant frequencies $k_{2} h$ are listed in Table II and plotted in Fig. 4 for three cases of $a / h$, the ratio of wire radius to wire half-length. (Recall that the propagation constant in air is $k_{2}=2 \pi f / c$ where $f$ is the frequency in Hertz and $c$ is the speed of light $\approx 3 \times 10^{8} \mathrm{~m} / \mathrm{s}$. Note that an even resonance number $n$ corresponds to anti-resonance for the impedance of a center-driven antenna.) The computations were carried out using MATLAB on a personal computer. Because several multiple integrals had to be evaluated many times, a running time of several hours was involved. 
TABLE II

COMPLEX VALUES OF $k_{2} h$ AT RESONANCE $n$ FOR THREE VALUES OF $a / h$

\begin{tabular}{|c|c|c|}
\hline$n$ & $a / h$ & $k_{2} h$ \\
\hline 1 & $10^{-4}$ & $0.956824-i 0.047401$ \\
\hline 2 & $10^{-4}$ & $1.934017-i 0.079959$ \\
\hline 3 & $10^{-4}$ & $2.905922-i 0.115751$ \\
\hline 4 & $10^{-4}$ & $3.872673-i 0.153152$ \\
\hline 5 & $10^{-4}$ & $4.837435-i 0.190228$ \\
\hline
\end{tabular}

\begin{tabular}{|c|c|c|}
\hline$n$ & $a / h$ & $k_{2} h$ \\
\hline 1 & $10^{-5}$ & $0.965436-i 0.038153$ \\
\hline 2 & $10^{-5}$ & $1.947398-i 0.063718$ \\
\hline 3 & $10^{-5}$ & $2.925628-i 0.091953$ \\
\hline 4 & $10^{-5}$ & $3.900158-i 0.121734$ \\
\hline 5 & $10^{-5}$ & $4.873157-i 0.151508$ \\
\hline
\end{tabular}

\begin{tabular}{|c|c|c|}
\hline$n$ & $a / h$ & $k_{2} h$ \\
\hline 1 & $10^{-6}$ & $0.970820-i 0.031885$ \\
\hline 2 & $10^{-6}$ & $1.955503-i 0.052866$ \\
\hline 3 & $10^{-6}$ & $2.937355-i 0.076062$ \\
\hline 4 & $10^{-6}$ & $3.916394-i 0.100618$ \\
\hline 5 & $10^{-6}$ & $4.894259-i 0.125246$ \\
\hline
\end{tabular}

First five complex resonant values of $k_{2} h$

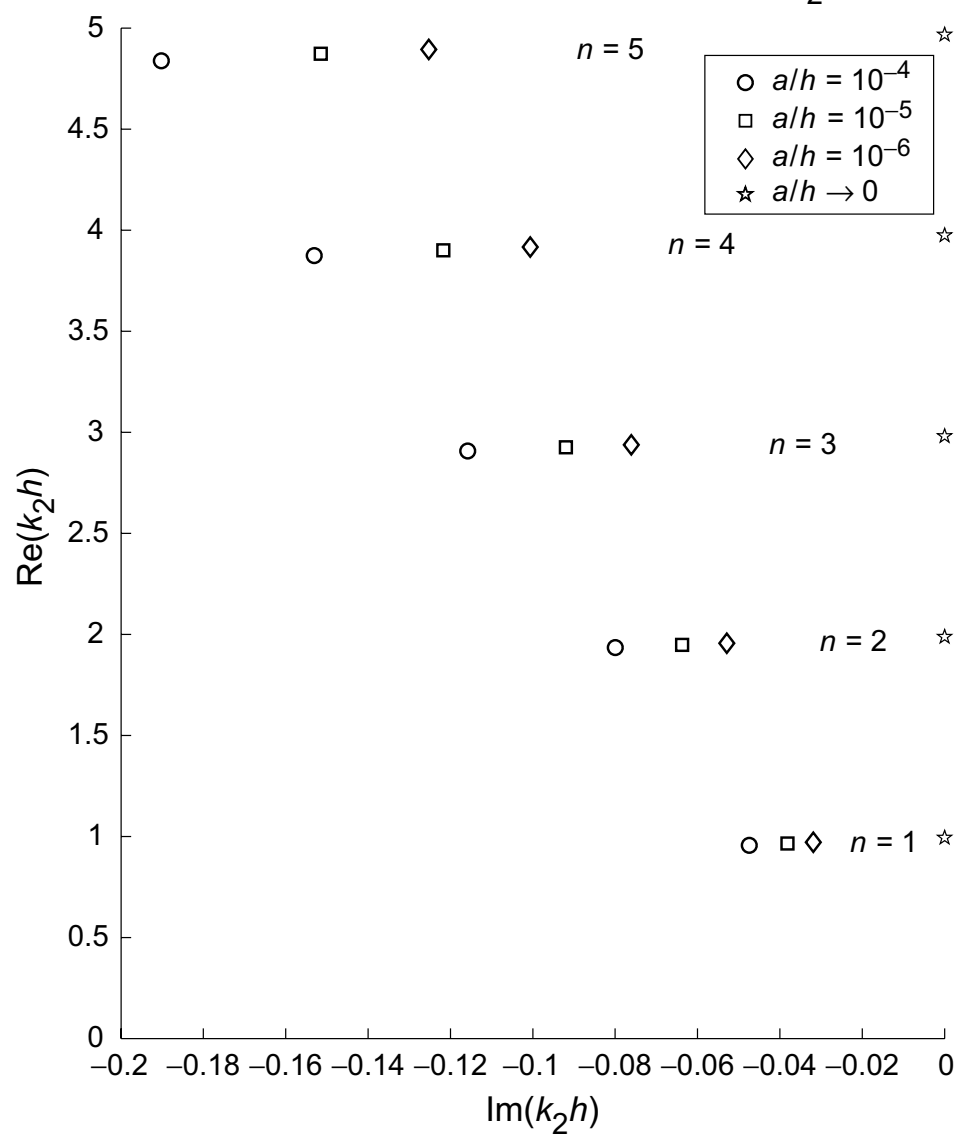

Fig. 4. Plot of first five resonant values of $k_{2} h$ for $k_{1} / k_{2}=2$.

\section{CONCLUSION}

The problem of determining the first few complex-valued resonant frequencies of a straight wire on a flat earth-air interface has been formulated and solved. The approach started from the electric-field kernel, which, however, contains a non-integrable singularity, evaded by finding the zero point in the Fourier transform of the electric field kernel. Using this zero point $k_{0}$ we arrived at the integro-differential equation (21), in which the kernel, defined by (37), is a double integral over a slowly decreasing, oscillating integrand. This kernel was transformed into a form suitable for numerical analysis. Although an exact determination of the resonant frequencies would require solving the extremely challenging integrodifferential equation (21), a variational technique allowed a crude approximation to the resonant current to 


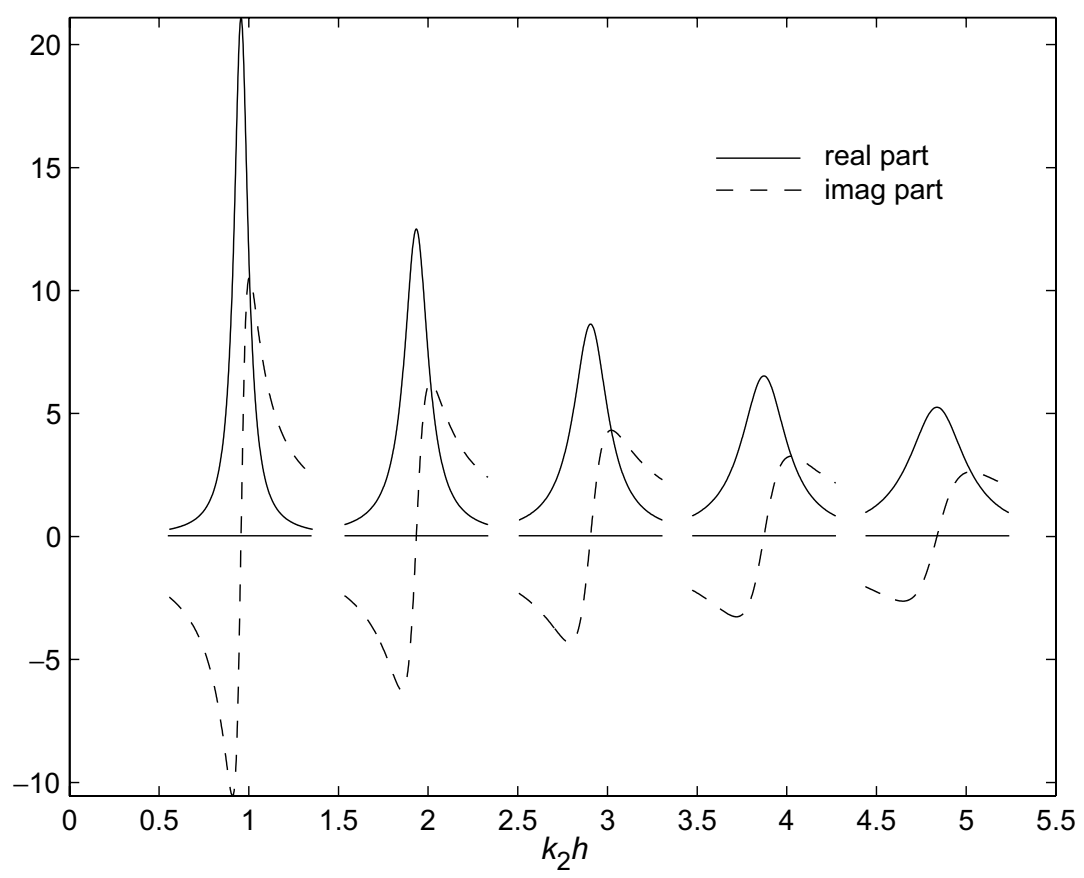

Fig. 5. Plot of $1 /\left(k_{2} h-k_{2, n}\right)$ for case of $k_{1} / k_{2}=2$ and $a / h=10^{-4}$.

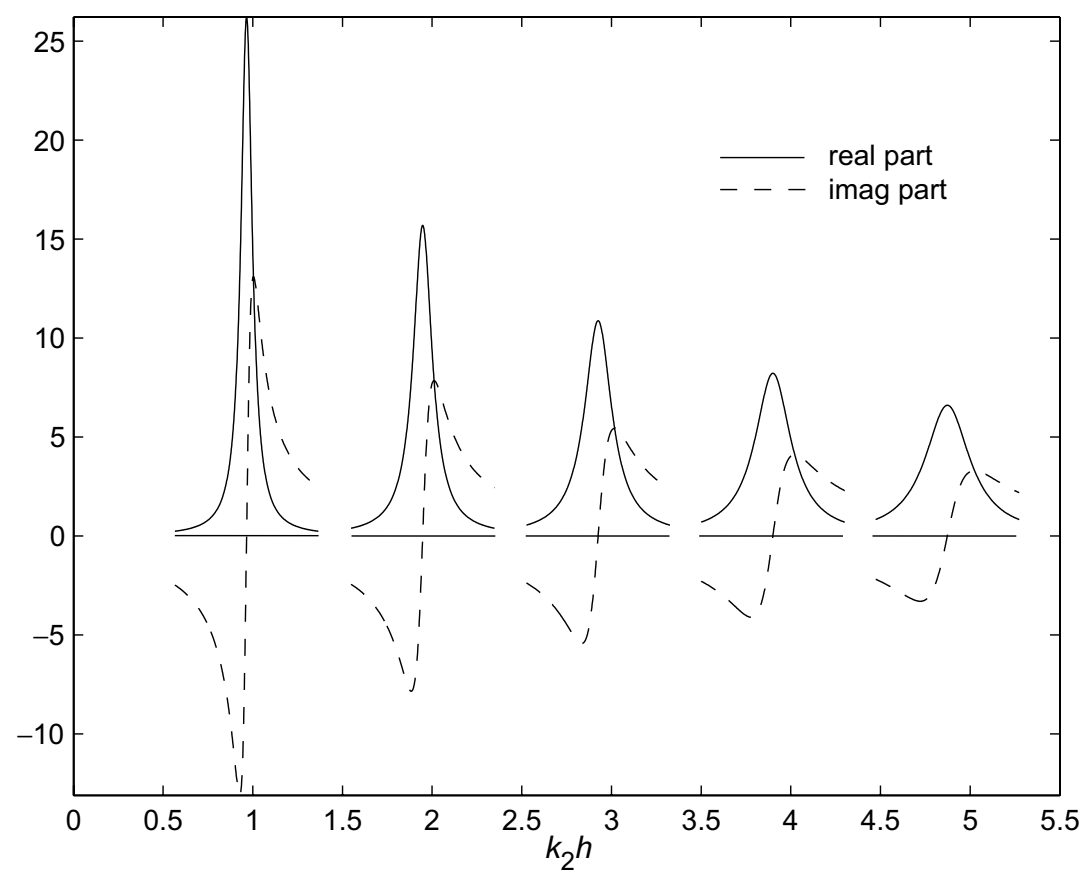

Fig. 6. Plot of $1 /\left(k_{2} h-k_{2, n}\right)$ for case of $k_{1} / k_{2}=2$ and $a / h=10^{-5}$.

be combined with our hard-won kernel to determine approximations to the resonant frequencies accurate to better than $0.1 \%$.

Because of the crude approximating current, we expect the method presented here to offer an accuracy in resonant frequencies on the order of $0.1 \%$ only for the first five resonances and for a ratio of wire diameter to wire length that is less than or equal to $10^{-4}$.

The resonant frequencies as determined here can enter a future analysis of backscattering by a wire on an earth-air interface. At frequencies near resonance, the backscattered field is approximately a product 


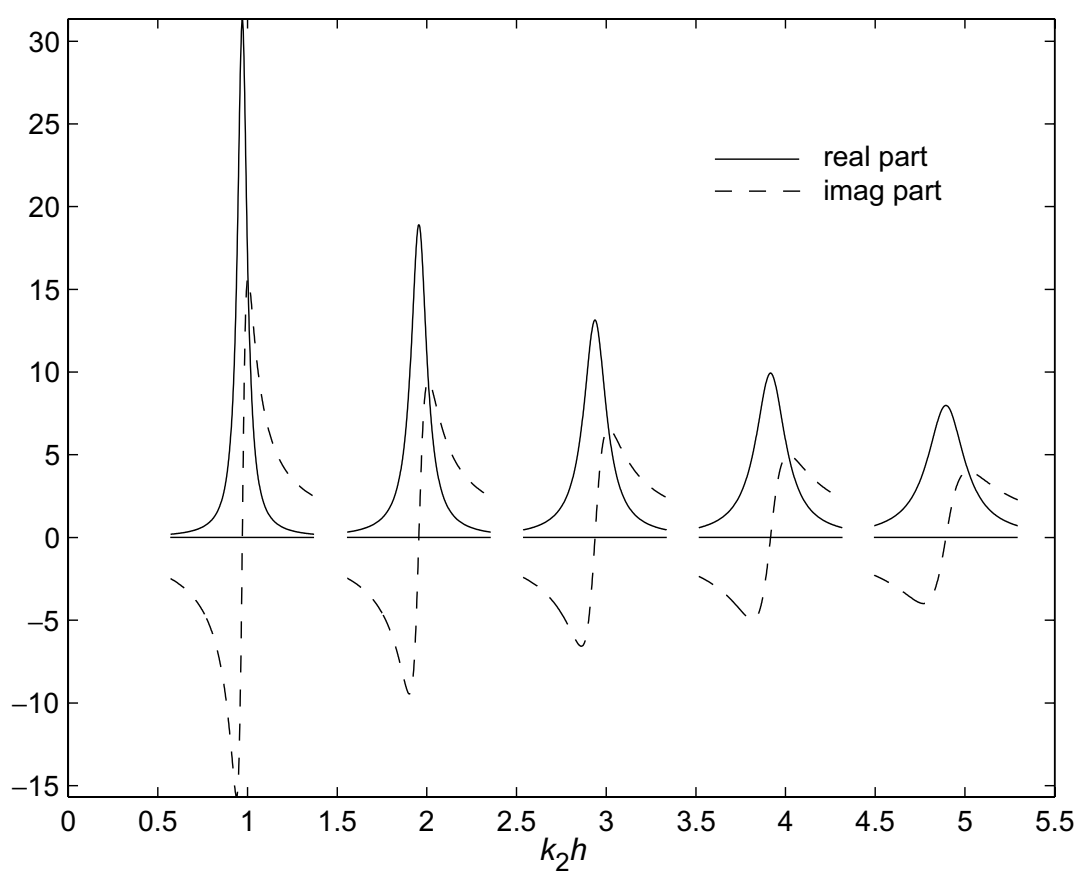

Fig. 7. Plot of $1 /\left(k_{2} h-k_{2, n}\right)$ for case of $k_{1} / k_{2}=2$ and $a / h=10^{-6}$.

of a geometric factor (involving the incident field and the resonant current) and a frequency factor; the frequency factor near the $n$-th resonance is proportional to $1 /\left(k_{2} h-k_{2, n}\right) \sim 1 /\left(\omega-\omega_{n}\right)$, the real and imaginary parts of which are plotted in Figs. 5, 6, and 7 for the same three cases of $a / h$ as reported in Table II. Also left to future analysis are the effects of: (1) departure of the wire from straightness; (2) departure of the earth-air interface from flatness; and (3) resistive loss in the wire.

\section{ACKNOWLEDGMENT}

This work was undertaken at the suggestion of James Hersey, whom we thank for posing the problem and for many helpful discussions along the way. We thank the referees for extraordinarily helpful suggestions.

\section{APPENDIX A \\ INTEGRATIONS FOR $I_{s n}$ AND $I_{c n}$}

Because $\boldsymbol{K}\left(x-x^{\prime}\right)$ depends only on the difference between $x$ and $x^{\prime}$, it must be possible to rewrite the integrals in (27) and (28) as single integrals. We do this without using the fact that $\boldsymbol{K}$ is an even function of its argument. Define

$$
I_{e}\left(\alpha, \alpha^{\prime}\right)=\int_{-h}^{h} d x e^{i \alpha x} \int_{-h}^{h} d x^{\prime} \boldsymbol{K}\left(x-x^{\prime}\right) e^{i \alpha^{\prime} x^{\prime}} .
$$

Changing integration variables $x \rightarrow-x^{\prime}$ and $x^{\prime} \rightarrow-x$ produces the relation

$$
I_{e}\left(-\alpha^{\prime},-\alpha\right)=I_{e}\left(\alpha, \alpha^{\prime}\right) .
$$

With this relation one expresses $I_{s n}$ and $I_{c n}$ as

$$
\begin{aligned}
& I_{s n}=-\frac{1}{4}\left[2 I_{e}\left(\kappa_{n}, \kappa_{n}\right)-I_{e}\left(\kappa_{n},-\kappa_{n}\right)-I_{e}\left(-\kappa_{n}, \kappa_{n}\right)\right], \\
& I_{c n}=\frac{1}{4}\left[2 I_{e}\left(\kappa_{n}, \kappa_{n}\right)+I_{e}\left(\kappa_{n},-\kappa_{n}\right)+I_{e}\left(-\kappa_{n}, \kappa_{n}\right)\right],
\end{aligned}
$$




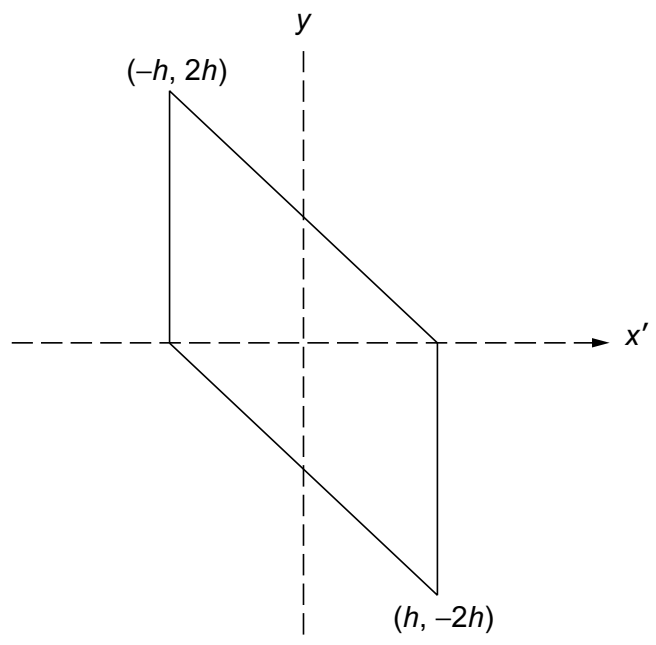

Fig. 8. Integration region on $\left(x^{\prime}, y\right)$-plane.

so that we have

$$
\begin{aligned}
I_{s n}+I_{c n} & =\frac{1}{2}\left[I_{e}\left(\kappa_{n},-\kappa_{n}\right)+I_{e}\left(-\kappa_{n}, \kappa_{n}\right)\right], \\
I_{s n}-I_{c n} & =-I_{e}\left(\kappa_{n}, \kappa_{n}\right) .
\end{aligned}
$$

For the reduction to single integrals we compute, using the integration region shown in Fig. 8,

$$
\begin{aligned}
I_{e}\left(\alpha, \alpha^{\prime}\right)= & \int_{-h}^{h} d x^{\prime} \int_{-h-x^{\prime}}^{h-x^{\prime}} d y e^{i \alpha\left(y+x^{\prime}\right)} e^{i \alpha^{\prime} x^{\prime}} \boldsymbol{K}(y) \\
= & \int_{-2 h}^{0} d y \boldsymbol{K}(y) e^{i \alpha y} \int_{-h-y}^{h} d x^{\prime} e^{i\left(\alpha+\alpha^{\prime}\right) x^{\prime}}+\int_{0}^{2 h} d y \boldsymbol{K}(y) e^{i \alpha y} \int_{-h}^{h-y} d x^{\prime} e^{i\left(\alpha+\alpha^{\prime}\right) x^{\prime}} \\
= & \frac{1}{i\left(\alpha+\alpha^{\prime}\right)}\left[\int_{-2 h}^{0} d y \boldsymbol{K}(y)\left(e^{i\left(\alpha+\alpha^{\prime}\right) h} e^{i \alpha y}-e^{-i\left(\alpha+\alpha^{\prime}\right) h} e^{-i \alpha^{\prime} y}\right)\right. \\
& \left.+\int_{0}^{2 h} d y \boldsymbol{K}(y)\left(e^{i\left(\alpha+\alpha^{\prime}\right) h} e^{-i \alpha^{\prime} y}-e^{-i\left(\alpha+\alpha^{\prime}\right) h} e^{i \alpha y}\right)\right] .
\end{aligned}
$$

The case $\alpha^{\prime}=-\alpha$ is worked out directly to show

$$
I_{e}(\alpha,-\alpha)=\int_{-2 h}^{0} d y \boldsymbol{K}(y)(2 h+y) e^{i \alpha y}+\int_{0}^{2 h} d y \boldsymbol{K}(y)(2 h-y) e^{i \alpha y}
$$

In the special case which we have here, in which $\boldsymbol{K}(y)=\boldsymbol{K}(-y)$ and $\kappa_{n}=n \pi / 2 h, I_{s n} \pm I_{c n}$ simplify to

$$
\begin{aligned}
& I_{s n}+I_{c n}=2 \int_{0}^{2 h} d y \boldsymbol{K}(y)(2 h-y) \cos \frac{n \pi y}{2 h} \\
& I_{s n}-I_{c n}=(-1)^{n} \frac{4 h}{n \pi} \int_{0}^{2 h} d y \boldsymbol{K}(y) \sin \frac{n \pi y}{2 h}
\end{aligned}
$$

which are the single integrals that we wanted to obtain. 


\section{REFERENCES}

[1] C. E. Baum, "The singularity expansion method," in Transient Electromagnetic Fields, L. B. Felsen, Ed. New York: Springer-Verlag, 1975.

[2] C. E. Baum, "Emerging technology for transient and broad-band analysis and synthesis of antennas and scatterers," Proc. IEEE, vol. 64, pp. 1598-1616, Nov. 1976.

[3] H. Kober, Dictionary of Conformal Representations. New York: Dover, 1957, p. 59.

[4] R. W. P. King, The Theory of Linear Antennas. Cambridge, MA: Harvard Univ. Press, 1956.

[5] F. M. Tesche, "On the analysis of scattering and antenna problems using the singularity expansion technique," IEEE Trans. Antennas Propag., vol. AP-21, pp. 53-62, Jan. 1973.

[6] J. M. Myers, S. S. Sandler, and T. T. Wu, "Electromagnetic resonances of a straight wire," IEEE Trans. Antennas Propag., vol. 59, pp. 129-134, Jan. 2011.

[7] R. W. P. King, M. Owens, and Tai Tsun Wu, Lateral Electromagnetic Waves: Theory and Applications to Communications, Geophysical Exploration and Remote Sensing. New York: Springer-Verlag, 1992.

[8] J. M. Myers, S. S. Sandler, and T. T. Wu, "Electromagnetic Resonances of a Wire on an Earth-Air Interface," Technical Report for Army Research Office Grant W911NF-07-1-0509, 12 November 2009, available from the Defense Technical Information Center, http://www.dtic.mil/ by searching on ADA520611.

[9] A. Erdélyi, Ed., Higher Transcendental Functions, vol. II. New York: McGraw-Hill, 1953.

[10] B. L. Coleman, "Propagation of electromagnetic disturbances along a thin wire in a horizontally stratified medium,” Phil. Mag. Series 7, vol. 41, pp. 276-288, 1950.

[11] J. R. Wait, "Theory of propagation along a thin wire parallel to an interface," Radio Science, vol. 7, pp. 675-679, 1972. 